\title{
Evaluating the Anticancer Activity of Hedyotis diffusa Water Extract Against Human Breast Cancer MCF7 Cells
}

\author{
Binbing Ling ${ }^{1, \$}$, Qiulin Dong ${ }^{1, \$}$, Wanpeng Sun ${ }^{1}$, Deborah Michel ${ }^{1}$, Rubin Jiao ${ }^{1}$, Ramaswami \\ Sammynaiken ${ }^{2,3}$, Yijiang Zhou ${ }^{4}$ and Jian Yang ${ }^{1, *}$
}

\author{
${ }^{1}$ Drug Discovery and Development Research Group, College of Pharmacy and Nutrition, University of Saskatchewan, \\ 110 Science Place, Saskatoon, SK S7N 5C9, Canada \\ ${ }^{2}$ Saskatchewan Structural Sciences Centre, University of Saskatchewan, 110 Science Place, Saskatoon, SK S7N 5C9, \\ Canada \\ ${ }^{3}$ Department of Biochemistry, University of Saskatchewan, 107 Wiggins Road, Saskatoon, SK S7N 5E5, Canada \\ ${ }^{4}$ School of Medicine, Zhejiang University, Hangzhou, Zhejiang 310058, P. R. of China
}

\begin{abstract}
Hedyotis diffusa has been used to treat cancers in traditional Chinese medicine for many years. Recently, several anthraquinone compounds were extracted from Hedyotis diffusa and identified as its anticancer ingredients. These compounds are able to induce apoptosis in several types of cancer cells. In this study, we showed that the anticancer activity of Hedyotis diffusa was highly selective, possessing high cytotoxicity towards human breast cancer MCF7 cells but almost no cytotoxicity against the mammary epithelial MCF12A cells. The Hedyotis diffusa water extract also elevated the energy metabolism in both MCF12A and MCF7 cells; but the ATP/ADP ratio was still maintained at about 1.0 in the MCF12A cells to avoid either apoptosis or uncontrolled proliferation. In addition, Sheshecaojing, a health product of $H e-$ dyotis diffusa marketed in China, gave a dose-dependent cytotoxic response against the MCF7 cells.
\end{abstract}

Keywords: cytotoxicity, energy metabolism, apoptosis, ATP/ADP ratio, herbal extraction.

\section{INTRODUCTION}

Traditional herbal medicine has existed for thousands of years, and herbs are still commonly used as therapeutic agents worldwide. A variety of herbal products have been proposed to possess anticancer or cancer inhibition properties [1-4]. Recently, herbal remedy has been emerging as a promising supplement to modern cancer therapies [5]. According to a study conducted by the Northern California Cancer Center, almost 13\% women with breast cancer use herbal remedies [6]. However, the clinical application of herbal medicines is significantly impeded due to insufficient information on their active ingredients as well as the underlying functional mechanisms in spite of recent developments in nutraceutical research.

Hedyotis diffusa is an herbal medicine widely used as an anti-inflammatory agent in traditional Chinese medicine [79]. Because inflammation is a crucial component of cancer progression [10], Hedyotis diffusa has been included in therapies against various types of cancer for a long time without even knowing its active ingredients [11-13]. The

\footnotetext{
*Address correspondence to this author at the Drug Discovery and Development Research Group, College of Pharmacy and Nutrition, University of Saskatchewan, 110 Science Place, Saskatoon, SK S7N 5C9, Canada;

Tel: +1-306-966-6361; Fax: +1-306-966-6377; E-mail: jian.yang@usask.ca "Equal contribution;

*Author to whom correspondence should be addressed
}

ethanol extract from Hedyotis diffusa was shown to induce cell apoptosis in the HT-29 human colon cancers [14]. Recently, several anthraquinone compounds were extracted from Hedyotis diffusa and claimed to be the active anticancer ingredients $[15,16]$. Both 2-hydroxy-3-methylanthraquinone (HMAQ) and 2-hydroxy-1-methoxyanthraquinone induced cell apoptosis in human lung adenocarcinoma SPC-1-A, breast cancer Bcap-37 and heptoma HepG2 cells via mitochondria-dependent caspase-3 activation [15]; and HMAQ promoted cell apoptosis in human breast cancer MCF7 cells through the endoplasmic reticulum mediated $\mathrm{Ca}^{2+} /$ calpain/ caspase-4 pathway [16].

Cancer cells predominantly rely on aerobic glycolysis instead of mitochondrial oxidative phosphorylation for their ATP generation. Targeting the energy metabolism in cancer cells has recently obtained more research attentions as a novel therapeutic method in treating breast cancers. It is still unclear whether the Hedyotis diffusa water and ethanol extracts, as well as the extracted anthraquinone compounds, possess any effects towards the energy metabolism in either normal or cancer cells. Thus, in the current study, we showed that the Hedyotis diffusa water extract increased the energy metabolism in both normal mammary epithelial MCF12A and the breast cancer MCF7 cells. However, the ATP/ADP ratio was still maintained at about 1.0 in the MCF12A cells, suggesting that the Hedyotis diffusa treatment was well regulated to avoid uncontrolled proliferation 
in MCF12A. In addition, the combination with other anticancer herbal medicines such as Scutellaria barbata might decrease the anticancer activity of Hedyotis diffusa.

\section{MATERIALS AND METHODS}

\section{Materials}

Dried Hedyotis diffusa was purchased from a traditional Chinese medicine store in Calgary, Alberta, Canada. Sheshecaojing (containing $5 \mathrm{~g}$ Hedyotis diffusa, $1 \mathrm{~g}$ Scutellaria barbata, $1 \mathrm{~g}$ American ginseng and $3 \mathrm{~g}$ sucrose in each $10 \mathrm{~g}$ package) was manufactured by Guangzhou Medicines and Health Products I/E Corporation (Guangzhou, Guangdong Province, China) and purchased from a Chinese herbal store in Saskatoon, Saskatchewan, Canada. Human mammary epithelial cell line MCF12A and breast cancer cell line MCF7 were purchased from Cedarlane Canada (Burlington, Ontario, Canada). The CytoTox $96{ }^{\circledR}$ Non-Radioactive Cytotoxicity Assay kit was purchased from Promega Corporation (Madison, Wisconsin, USA).

\section{Extract Preparation}

The Hedyotis diffusa water extract was prepared by boiling $1 \mathrm{~g}$ of chopped Hedyotis diffusa in $100 \mathrm{~mL}$ deionized water for $1 \mathrm{hr}$. The solution was allowed to cool down at room temperature for at least $2 \mathrm{hr}$ before the top supernatant was used for cell treatments. For convenience, the extract solution was defined as concentration of $10 \mathrm{~g} / \mathrm{L}$ (i.e. $10 \mathrm{~g}$ of Hedyotis diffusa boiled in $1 \mathrm{~L}$ of water). The extract solution of $5 \mathrm{~g} / \mathrm{L}$ was prepared by diluting the above preparation with deionized water. The Sheshecaojing solution $(40 \mathrm{~g} / \mathrm{L})$ was prepared by boiling 1 package $(10 \mathrm{~g})$ of Sheshecaojing in $250 \mathrm{~mL}$ water for $1 \mathrm{hr}$. The other Sheshecaojing solutions (concentrations from $0.31 \mathrm{~g} / \mathrm{L}$ to $20 \mathrm{~g} / \mathrm{L}$ ) were prepared by serial water dilution of the above preparation.

\section{Cell Culture and Cytotoxicity Assay}

Both MCF12A and MCF7 cells were cultured in T-75 cell culture flasks under the recommended culture conditions by Cedarlane Canada. The cells were incubated under a humidified, $5 \% \mathrm{CO}_{2}$ atmosphere at $37^{\circ} \mathrm{C}$ with the culture media changed every 2-3 days. The cells were then collected from the T-75 cell culture flasks, re-suspended in appropriate culture media, and plated in 96-well culture plates with each well containing about $5 \times 10^{4}$ cells. The cells were allowed to grow for another day before being treated with either the Hedyotis diffusa water extract or Sheshecaojing solution for $24 \mathrm{hr}$. The cytotoxicity was measured using the CytoTox $96^{\circledR}$ Non-radioactive Cytotoxicity Assay kit from Promega Corporation. Cells treated with the culture media were used as the negative controls. The cytotoxicity was calculated using the following equation:

\section{Cytetoxicity (\%) $=\frac{\text { Experimental - Control }}{\text { Maximum (Lysis) - Control }}$}

\section{ATP and ADP Measurements}

The concentrations of ATP and ADP in Hedyotis diffusa treated MCF12A and MCF7 cells (maintained at least $80 \%$ confluence after treatment) were measured with HPLC-UV method as described by Olkowski et al. [17]. Briefly, the cells were quickly washed with $1 \mathrm{~mL}$ phosphate buffered saline (PBS) twice after $24 \mathrm{hr}$ treatment. Ice cold perchloric acid $(0.35 \mathrm{~mL}$ of $0.7 \mathrm{M})$ was added into each well. The cells were detached from the plate using a cell scrubber. The cell mixtures were then homogenized and centrifuged at 12000 rpm for $5 \mathrm{~min}$. The supernatants were collected and neutralized with $2 \mathrm{M}$ potassium hydroxide to bring $\mathrm{pH}$ close to 7.0. The supernatants were subsequently filtered through $0.45 \mu \mathrm{m}$ filter, and $10 \mu \mathrm{L}$ of each sample was injected into a C-18 column using gradient. Two mobile phase components used included $20 \mathrm{mM}$ potassium phosphate buffer $(\mathrm{pH} 7.0)$ and $100 \%$ methanol. The gradient was $100 \%$ phosphate buffer from $0-6.5 \mathrm{~min}, 100 \%$ methanol from $6.5-12.5 \mathrm{~min}$, and $100 \%$ phosphate buffer from 12.5 - 25 min for column reequilibration, which was sufficient to achieve stable baseline conditions. The ATP and ADP levels in the samples were monitored at $210 \mathrm{~nm}$.

\section{RESULTS AND DISCUSSION}

\section{Selective Anticancer Activity Against Human Breast can- cer MCF7 Cells}

The cytotoxicity of the Hedyotis diffusa water extract was measured towards human mammary epithelial MCF12A and breast cancer MCF7 cell lines under two different concentrations (Fig. 1). The water extract did not exhibit any cytotoxicity against the mammary epithelial MCF12A cells under either concentration. However, it showed $13 \%$ cytotoxicity at concentration of $5 \mathrm{~g} / \mathrm{L}$ and $45 \%$ cytotoxicity at concentration of $10 \mathrm{~g} / \mathrm{L}$, respectively, against the breast cancer MCF7 cells. This indicated that the anticancer activity of Hedyotis diffusa is highly selective. Since no cytotoxicity was observed even at concentration of $10 \mathrm{~g} / \mathrm{L}$ in the MCF12A cells, the Hedyotis diffusa water extract may be pushed to much higher concentrations in order to achieve better cytotoxic effect against the breast cancer MCF7 cells. Although direct administration of pure HMAQ $(>20 \mu \mathrm{M})$ induced apoptosis in the MCF7 cells [16], further studies are definitely required to answer the following two questions. The first question is whether HMAQ can induce apoptosis in the MCF7 cells under its naturally occurring concentrations in Hedyotis diffusa; and the second question is whether any

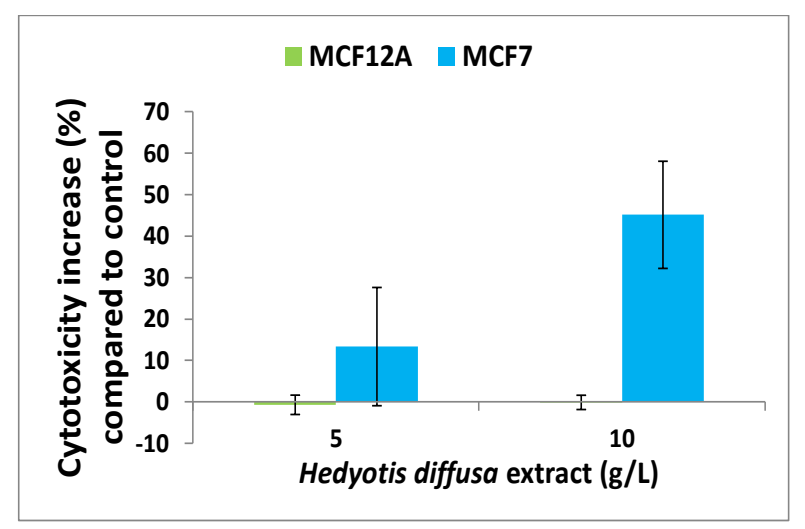

Fig. (1). Cytotoxicity (\%) of the Hedyotis diffusa water extract in human mammary epithelial MCF12A cells (shown in green) and breast cancer MCF7 cells (shown in blue). 
other ingredients such as flavonoids are also involved in the apoptotic effect of Hedyotis diffusa.

\section{Effects on Energy Metabolism}

Cell growth and function are closely associated with the cellular energy production. Thus, the effect of the Hedyotis diffusa water extract on energy metabolism was investigated in both MCF12A and MCF7 cells. As shown in Fig. (2a), the ATP level was increased by about 2.8 folds in the MCF12A cells. Since normal differentiated cells rely on mitochondrial oxidative phosphorylation (producing 36 ATPs per glucose molecule) for their energy production [18, 19], Hedyotis diffusa is likely increasing the mitochondrial oxidative phosphorylation in the MCF12A cells. However, the ATP/ADP ratio was still maintained at about 1.0 (Fig. 2b), indicating that the Hedyotis diffusa-treated MCF12A cells were well regulated by cellular signaling pathways to avoid uncontrolled proliferation. In the MCF7 cells, the ATP level was elevated by 3.6 folds (Fig. 2a). Because cancer cells predominantly rely on aerobic glycolysis for their ATP generation (producing 2 ATPs and 1 lactate per glucose molecule), the higher ATP level in the MCF7 cells might be due to increased share of the mitochondrial oxidative phosphorylation (as observed in the MCF12A cells) in the total energy metabolism rather than elevated aerobic glycolysis. One of the benefits of aerobic glycolysis to the cancer cells is to increase their biomass in preparation for proliferation [19].

(a)

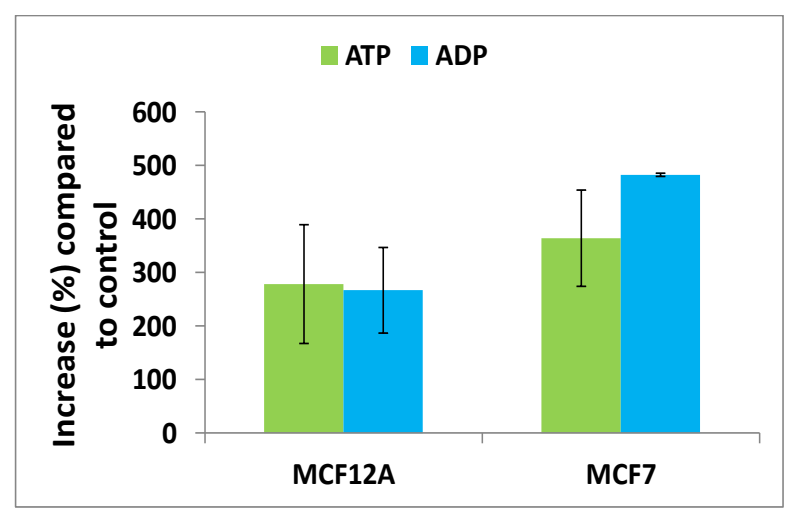

(b)

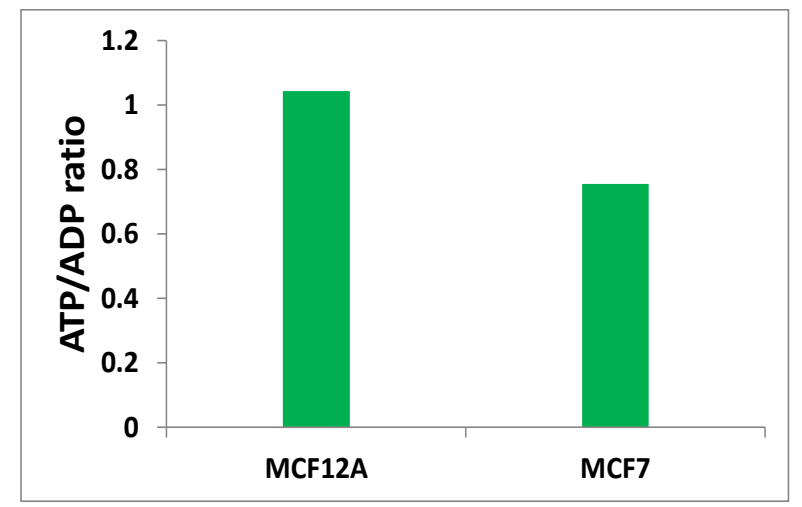

Fig. 2. (a) ATP and ADP levels in the MCF12A and MCF7 cells after Hedyotis diffusa treatment for 24 hrs. (b) ATP/ADP ratio in the treated MCF12A and MCF7 cells.
Therefore, the increase in mitochondrial oxidative phosphorylation may not be favorable for the MCF7 cells as it will decrease the biomass accumulation as well as the proliferation in the MCF7 cells. Furthermore, the ATP/ADP was reduced to 0.75 in the MCF7 cells after the Hedyotis diffusa treatment (Fig. 2b), suggesting that the cells were undergoing apoptosis. Although the identity of the compounds that may increase mitochondrial oxidative phosphorylation in the cells is unclear, one potential candidate is the iridoid glycosides, which have been identified as another family of active ingredients in Hedyotis diffusa [20,21] and shown to ameliorate mitochondrial energy metabolism in rat cortical neurons experiencing oxygen-glucose deprivation [22].

\section{Anticancer Activity of Sheshecaojing}

Single herbal remedy is quite rare in traditional Chinese medicine. Hedyotis diffusa is usually used in combination with Scutellaria barbata. Sheshecaojing, consisting of 50\% Hedyotis diffusa, 10\% Scutellaria barbata, 10\% American ginseng and $30 \%$ sucrose, is marketed as an anti-inflammatory health product in China. Since both Scutellaria barbata and American ginseng possess anticancer activities [2326], Sheshecaojing was expected to exhibit better cytotoxic effect against the cancer cells than Hedyotis diffusa. In Fig. (3), Sheshecaojing was shown to impose little cytotoxicity to the MCF12A cells; however, it gave an almost dosedependent cytotoxic response in the MCF7 cells. The cytotoxicity was $32 \%$ at concentration of $20 \mathrm{~g} / \mathrm{L}$ for Sheshecaojing (equivalent to $10 \mathrm{~g} / \mathrm{L}$ of Hedyotis diffusa), which was much less than that of $45 \%$ for Hedyotis diffusa alone. This decrease in cytotoxicity might be due to the addition of sucrose since sucrose is generally believed to diminish or deplete herbal functions in traditional Chinese medicine. However, the cytotoxicity for Sheshecaojing was $26 \%$ at concentration of $10 \mathrm{~g} / \mathrm{L}$ (equivalent to $5 \mathrm{~g} / \mathrm{L}$ of Hedyotis diffusa), which was $13 \%$ higher than that for Hedyotis diffusa alone. Therefore, further studies are needed to clarify the influences of Scutellaria barbata, American ginseng and sucrose on the anticancer activity of Hedyotis diffusa.

\section{CONCLUSIONS}

Hedyotis diffusa is an herbal medicine that has been used to treat cancers in traditional Chinese medicine for a very

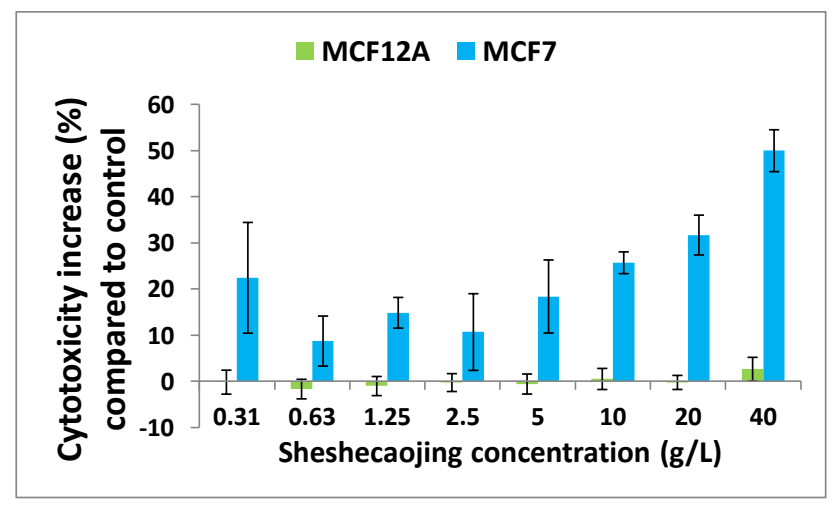

Fig. (3). Cytotoxicity (\%) of Sheshecaojing against human mammary epithelial MCF12A cells (shown in green) and breast cancer MCF7 cells (shown in blue). 
long period of time. In this study, we showed that Hedyotis diffusa, as well as the commercial health product Sheshecaojing, exhibited highly selective cytotoxicity against human breast cancer MCF7 cells. The Hedyotis diffusa water extract stimulated the ATP generation in both normal mammary epithelial MCF12A and breast cancer MCF7 cells possibly via increasing the mitochondrial oxidative phosphorylation. However, the increase in mitochondrial oxidative phosphorylation may not be beneficial to the MCF7 cells.

\section{CONFLICT OF INTEREST}

The authors confirm that this article content has no conflicts of interest.

\section{ACKNOWLEDGEMENT}

This work was partially supported by a research grant from the Canadian Breast Cancer Foundation.

\section{REFERENCES}

[1] Li, G.; Sepkovic, D.W.; Bradlow, H.L.; Telang, N.T.; Wong, G.Y. Lycium barbarum inhibits growth of estrogen receptor positive human breast cancer cells by favorably altering estradiol metabolism. Nutr. Cancer, 2009, 61, 408-414.

[2] Lesiak, K.; Koprowska, K.; Zalesna, I.; Nejc, D.; Düchler, M.; Czyz, M. Parthenolide, a sesquiterpene lactone from the medical herb feverfew, shows anticancer activity against human melanoma cells in vitro. Melanoma Res., 2010, 20, 21-34.

[3] He, Z.H.; Ge, W.; Yue, G.G.; Lau, C.B.; He, M.F.; But, P.P. Antiangiogenic effects of the fruit of Alpinia oxyphylla. J. Ethnopharmacol., 2010, 132, 443-449.

[4] Kim, H.J.; Kim, J.C.; Min, J.S.; Kim, M.J.; Kim, J.A.; Kor, M.H.; Yoo, H.S.; Ahn, J.K. Aqueous extract of Tribulus terrestris Linn induces cell growth arrest and apoptosis by down-regulating NF- $\mathrm{kB}$ signaling in liver cancer cells. J. Ethnopharmacol., 2011, 136, 197203.

[5] Engdal, S.; Steinsbekk, A.; Klepp, O.; Nilsen, O.G. Herbal use among cancer patients during palliative or curative chemotherapy treatment in Norway. Support. Care Cancer, 2008, 16, 763-769.

[6] Lee, M.M.; Lin, S.S.; Wrensch, M.R.; Adler, S.R.; Eisenberg, D. Alternative therapies used by women with breast cancer in four ethnic populations. J. Natl. Cancer Inst., 2000, 92, 42-47.

[7] Ahmad, R.; Ali, A.M.; Israf, D.A.; Ismail, N.H.; Shaari, K. Lajis, N.H. Antioxidant, radical-scavenging, anti-inflammatory, cytotoxic and antibacterial activities of methanolic extracts of some Hedyotis species. Life Sci., 2005, 76, 1953-1964.

[8] Lin, C.C.; Ng, L.T.; Yang, J.J.; Hsu, Y.F. Anti-inflammatory and hepatoprotective activity of peh-hue-juwa-chi-cao in male rats. Am. J. Chin. Med., 2002, 30, 225-234.

[9] Shan, B.E., Yoshida, Y.; Sugiura, T.; Yamashita, U. Stimulating activity of Chinese medicinal herbs on human lymphocytes in vitro. Int. J. Immunopharmacol., 1999, 21, 149-159.

[10] Coussens, L.M.; Werb, Z. Inflammation and cancer. Nature, 2002, 420, 860-867.
[11] Liao, L.L.; Chen, C.H.; Chen, G.C. Formosan medicinal herb, Hedyotis diffusa Willd, as an antitumor agent. Taiwan Yi Xue Hui Za Zhi, 1979, 78, 658-660.

[12] Wong, B.Y.; Lau, B.H.; Jia, T.Y.; Wan, C.P. Oldenlandia diffusa and Scutellaria barbata augment macrophage oxidative burst and inhibit tumor growth. Cancer Biother. Radiopharm., 1996, 11, 5156.

[13] Gupta, S.; Zhang, D.; Yi, J.; Shao, J. Anticancer activities of Oldenlandia diffusa. J. Herb. Pharmacother., 2004, 4, 21-33.

[14] Lin, J.; Chen, Y.; Wei, L.; Chen, X.; Xu, W.; Hong, Z.; Sferra, T.J.; Peng, J. Hedyotis diffusa WILLD extract induces apoptosis via activation of the mitochondrion-dependent pathway in human colon carcinoma cells. Int. J. Oncol., 2010, 37, 1331-1338.

[15] Shi, Y.; Wang, C.H.; Gong, X.G. Apoptosis-inducing effects of two anthraquinones from Hedyotis diffusa WILLD. Biol. Pharm. Bull., 2008, 31, 1075-1078.

[16] Liu, Z.; Liu, M.; Liu, M.; Li, J. Methylanthraquinone from $\mathrm{He}$ dyotis diffusa WILLD induces $\mathrm{Ca}(2+)$-mediated apoptosis in human breast cancer cells. Toxicol. In Vitro, 2010, 24, 142-147.

[17] Olkowski, A.A.; Nain, S.; Wojnarowicz, C.; Laarveld, B.; Alcorn, J.; Ling, B.B. Comparative study of myocardial high energy phosphate substrate content in slow and fast growing chicken and in chickens with heart failure and ascites. Comp. Biochem. Physiol., Part A Mol. Integr. Physiol., 2007, 148, 230-238.

[18] Vander Heiden, M.G.; Cantley, L.C.; Thompson, C.B. Understanding the Warburg effect: the metabolic requirements of cell proliferation. Science, 2009, 324, 1029-1033.

[19] Johannsen, D.L.; Ravussin, E. The role of mitochondria in health and disease. Curr. Opin. Pharmacol., 2009, 9, 780-786.

[20] Zhang, Y.; Chen, Y.; Fan, C.; Ye, W.; Luo, J. Two new iridoid glucosides from Hedyotis diffusa. Fitoterapia, 2010, 81, 515-517.

[21] Li, C.; Xue, X.; Zhou, D.; Zhang, F.; Xu, Q.; Ren, L.; Liang, X. Analysis of iridoid glucosides in Hedyotis diffusa by highperformance liquid chromatography/electrospray ionization tandem mass spectrometry. J. Pharm. Biomed. Anal., 2008, 48, 205-211.

[22] Jiang, W.L.; Fu, F.H.; Zheng, S.G.; Zhang, D.L.; Zhu, H.B.; Hou, J. 8-O-acetyl shanzhiside methylester attenuates apoptosis and ameliorates mitochondrial energy metabolism in rat cortical neurons exposed to oxygen-glucose deprivation. Eur. J. Pharmacol., 2010, 629, 20-24.

[23] Marconett, C.N.; Morgenstern, T.J.; San Roman, A.K.; Sundar, S.N.; Singhal, A.K.; Firestone, G.L. BZL101, a phytochemical extract from the Scutellaria barbata plant, disrupts proliferation of human breast and prostate cancer cells through distinct mechanisms dependent on the cancer cell phenotype. Cancer Biol. Ther., 2010, 10, 397-405.

[24] Dai, Z.J.; Wang, X.J.; Li, Z.F.; Ji, Z.Z.; Ren, H.T.; Tang, W.; Liu, X.X.; Kang, H.F.; Guan, H.T.; Song, L.Q. Scutellaria barbate extract induces apoptosis of hepatoma H22 cells via the mitochondrial pathway involving caspase-3. World J. Gastroenterol., 2008, 14, 7321-7328.

[25] Peralta, E.A.; Murphy, L.L.; Minnis, J.; Louis, S.; Dunnington, G.L. American Ginseng inhibits induced COX-2 and NFKB activation in breast cancer cells. J. Surg. Res., 2009, 157, 261-267.

[26] King, M.L.; Murphy, L.L. American ginseng (Panax quinquefolius L.) extract alters mitogen-activated protein kinase cell signaling and inhibits proliferation of MCF-7 cells. J. Exp. Ther. Oncol., 2007, 6, 147-155. 